\title{
Akademische und Offizielle Statistik vereint. Ein Interview mit Peter Hackl
}

\author{
Peter Hackl \\ Vormals Statistik Austria, WU Wien
}

\author{
Werner Müller \\ JKU Linz
}

\author{
Matthias Templ \\ TU Wien \& Statistics Austria
}

\begin{abstract}
Das Interview mit Peter Hackl wurde von Werner Müller und Matthias Templ am 20.03.2014 gehalten. Es zeichnet ein Bild des beruflichen Werdeganges von Peter Hackl, von der Physik und des Welthandeles mit der Statistik in der richtigen Skala, long runners bei Marathons und Textbüchern, von seiner Zeit an der Wirtschaftsuniversität in Wien, Abstechern zur „Handelshögskolan“, nach Abu Dhabi, und Peer-Reviews für Institutionen wie Eurostat. Weiters werden seine zahlreichen Führungsrollen in der ÖSG und in der Statistik Austria beleuchtet. Im Blickfeld ist auch eine Diskussion über die Ausrichtung der Statistik.
\end{abstract}

Peter Hackl, ist am 18 August 1942 in Linz geboren. Er war unter anderem Fachstatistischer Generaldirektor der Statistik Austria, Präsident der Österreichischen Statistischen Gesellschaft und Universtätsprofessor an der Wirtschaftuniversität Wien. Er hat mehrere Bücher veröffentlicht, wie z.B. „Einführung in die Ökonometrie" (Pearson Studium).

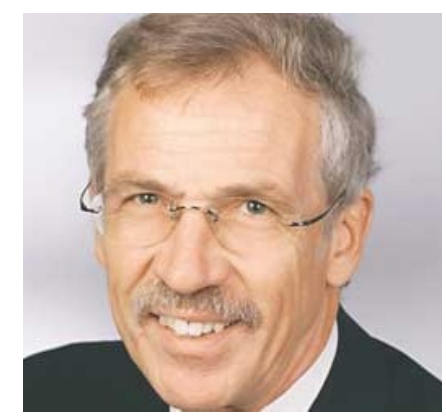

Keywords: Interview, Offizielle Statistik, Politikberatung.

Matthias Templ: Wir leben eine gute Tradition. Zwei ehemaligen Präsidenten der Statistischen Gesellschaft wurden bereits für das Austrian Journal of Statistics interviewed, du bist schon der Dritte in der Interviewreihe. Vielen Dank für deine Zusage.

Peter Hackl: Ich danke auch euch. Ihr habt mir ein sehr ehrenvolles Angebot gemacht, auf das es nicht ganz einfach war, ja oder nein zu sagen.

Werner Müller: Das Erste, was in deiner Biografie auffällt: du bist ein gebürtiger OÖer, Linzer, und hast in Wien studiert.

Peter Hackl: Es war eine Folge der Kriegsumstände, dass ich in Linz zur Welt gekommen bin. Meine Großeltern väterlicherseits waren aus dem Hausruck, und dort bin ich die ersten drei Jahre meines Lebens aufgewachsen. Einen Teil dieser Zeit lebte ich auch in 
Innsbruck. Ich erzähle ganz gern, dass ich Linzer bin. Tatsache ist aber, dass ich fast mein ganzes Leben in Wien verbrachte.

Werner Müller: Was natürlich noch auffällt ist, dass du ein Studium der techn. Physik an der TU Wien gemacht und nach dem Studium das Fach gewechselt hast.

\section{Peter Hackl:}

Das stimmt so nicht ganz. Beide Fächer, sowohl die Statistik als auch die Physik, kann man als Disziplin der angewandten Mathematik sehen. Ich habe mir in Mathematik immer sehr leicht getan, in der Schule schon und auch im Studium. Daher hat das Studium sehr gut gepasst.

In der ersten Vorlesung der Experimentalphysik, zentrales Fach für die jungen Studierenden der Physik, ist der honorige Professor Regler in den Hörsaal gekommen, hat in den übervollen Saal geschaut - es gab ca. 200 Physikanfänger - und hat gesagt: „Ich kann Ihnen sagen, es werden nicht mehr von Ihnen als die Zahl der Finger einer Hand einen Job als Physiker bekommen“. Es war dann nicht ganz so radikal, wie er das gesagt hat, aber es sind sehr viele meiner Studienkollegen nicht in der Physik geblieben; zum Teil sind sie auch im Ausland gelandet. Viele Studienkollegen haben sich der damals aufkommenden Informatik zugewendet. Statistik war damals kein populäres Fach. Mein Semesterkollege Peter Bauer hat sich im Rahmen seiner Dissertation mit einer statistischen Fragestellung befasst und ist so zur Biometrie gekommen. Ich habe während meiner Tätigkeit als Dissertant Paukerkurse in Statistik für WU-Studierende gehalten, damals noch an der Hochschule für Welthandel. Als ich mit meiner Doktorarbeit fertig wurde, ist eine Assistentenstelle am damals ziemlich neuen Statistikinstitut frei geworden. Ich bin in die Sprechstunde zu Professor Roppert gegangen und hab ihm meinen Werdegang geschildert einschließlich meiner Tätigkeit als Pauker.

Werner Müller: Und er hat dich ein Integral lösen lassen?

Peter Hackl: Nein (lacht). Professor Roppert hat mich nach zwei Tagen angerufen und ich habe dann einen Job gehabt, Assistent an der Welthandel. Das war im Sommer 1970.

Werner Müller: Das waren die relativen Anfänge des Instituts.

Peter Hackl: vorher ist es gegründet worden. Es war damals die Zeit, wo die Orientierung des Instituts in Richtung Statistik noch nicht sehr ausgeprägt war. Das Institut vermittelte den Studierenden eine Grundausbildung in Mathematik und in Statistik; daneben führte das Institut das Rechenzentrum der Welthandel. Mitarbeiter von Roppert waren Heinz Skala, der nach Berlin ging, und dessen Posten ich bekam, und Wolfgang Janko, inzwischen Emeritus des Department of Information Systems and Operations der Wirtschaftsuniversität.

Werner Müller: Und Professor Derflinger ist ja erst später gekommen.

Peter Hackl: Ja, er dürfte 1975 oder 1976 von der Uni Linz nach Wien gekommen sein und hat eine Abteilung des Instituts mit Schwerpunkt angewandte Statistik und Datenanalyse aufgebaut. Derflinger war gut in der Faktorenanalyse ausgewiesen. Die Abteilung Roppert befasste sich mit Themen in den Bereichen statistische Methoden, Wirtschaftsstatistik, operations research und Informatik.

Werner Müller: War ein bisschen verpönt mit Daten...

Peter Hackl: Im Statistik-Institut sind sehr unterschiedliche Themen behandelt worden; als Assistent hat man viele Freiheiten gehabt in diesem Institut. Professor Roppert hat es großartig verstanden, seine Mitarbeiter zu motivieren, und es wurden international herzeigbare Arbeiten produziert. Roppert konnte sehr gut das Gefühl vermitteln, dass gute Forschungsleistungen Voraussetzung einer erfolgreichen Karriere an der Uni sind, und es haben sich alle daran gehalten. Roppert hat seine Mitarbeiter auch sehr gefördert. 
Werner Müller: Die große Herausforderung waren ja die großen Studentenmengen. Die zu bewältigen und dafür eine sinnvolle Didaktik zu entwickeln, das war alles etwas Neuland; die Statistik- und Mathematikausbildung war ja komplett in eurer Hand.

Peter Hackl: Ja, die war in unserer Hand. Ich kann mich an mein erstes Jahr an der WU erinnern: In den Proseminaren sind Studierende, die keinen Sitzplatz mehr fanden, dicht gedrängt auf den Stiegen des Audi Max gesessen. In meiner Zeit als Student und auch später gab es in der heutigen Zeit unvorstellbare Zustände. Zu den Vorlesungen gab es kaum begleitende Literatur: Passende und studierfreundliche Lehrbücher waren kaum auf dem Markt; es gab keine Skripten; auf beharrliche Nachfrage wurden oft gänzlich ungeeignete Bücher empfohlen. Weil ich aus eigener Erfahrung gewusst habe, wie schwierig es die Studierenden haben, habe ich dann angefangen, den Studierenden zur Vorbereitung auf die Proseminare hektographierte Beispielsammlungen auszuteilen, aus denen dann bei der ÖH verlegte Skripten wurden.

Werner Müller: So sind dann die legendären Textbücher Hackl, Katzenbeisser, Panny entstanden.

Peter Hackl: Ja, so hat es angefangen.

Werner Müller: In wievielen Auflagen sind diese Textbücher erschienen?

Peter Hackl: Unzählige, das war Policy des Oldenbourg-Verlags. Die Statistik hat es auf elf, die Mathematik auf acht Auflagen gebracht.

Matthias Templ: Du hast erwähnt, dass die Ausrichtung des "Roppert'schen" Statistikinstituts relativ breit war. Das charakterisiert deine Forschungsausrichtung ebenfalls. Du bist sehr vielseitig und breit aufgestellt. Unter anderem hast dich beispielsweise auch früh mit Partial Least Squares Methoden beschäftigt.

Peter Hackl: Ich habe viel über lineare Modelle gearbeitet. Ich habe das Glück gehabt hab, dass ich ziemlich am Anfang meiner Assistententätigkeit zu einem Gastaufenthalt an die University of Edinburgh gekommen bin, wo Peter Fisk am Statistikinstitut gearbeitet hat. Er war einer der ersten, der über Mehrgleichungssysteme in der Ökonometrie gearbeitet und darüber auch ein Buch geschrieben hat. Ich war zwei Sommer für jeweils zwei Monate in Edinburgh. Peter Fisk hat mich auf ein interessantes Thema aufmerksam gemacht: Jim Durbin hat gemeinsam mit zwei Autoren vom britischen Statistikamt eine Untersuchung über die Identifikation von Brüchen in Regressionsmodellen gemacht, eine methodische Arbeit mit Anwendungen auf bekannte Datensätze. Es gab einen Vortrag über diese Arbeit vor der Royal Statistical Society und einen diskutierten Beitrag in der Serie B des Journal of the Royal Statistical Society, der 1975 erschienen ist. Peter Fisk war Diskutant dieses Beitrages und er hat mir einen Vorabdruck des Artikels gegeben. Von den Verfahren von Durbin \& Co ausgehend, habe ich eigene Ideen zur Diagnose von Strukturbrüchen entwickelt. Eines der Verfahren, die Durbin \& Co vorgeschlagen haben, basiert auf kumulativen Summen von sogenannten rekursiven Residuen, das sind im Wesentlichen one step ahead forecast errors. Technisch unkompliziertere Indikatoren basieren auf gleitenden Summen (moving sums) von rekursiven Residuen, deren Verteilungseigenschaften einfachere Tests erlauben. Entsprechende Verfahren waren der Kern meiner Habilschrift.

Werner Müller: Es sind zwei Papers entstanden, mit Peter Bauer.

Peter Hackl: Tests auf Strukturbrüche sind wichtige Instrumente für die Diagnostik von Regressionsmodellen, etwa zur Anwendung in der Ökonometrie, wo bei der Analyse von ökonomischen Zeitreihen immer die Frage zu klären ist, ob das Modell richtig spezifiziert ist, insbesondere, ob die Modellparameter über den gesamten Zeitraum als konstant angesehen werden können. Eine naheliegende Anwendungen dieser Idee in der Prozesskontrolle erlaubt es zu entscheiden, ob die Prozessvariable einen gewünschten Niveauwert 
einhält oder ob Abweichungen es notwendig machen, in den Prozess einzugreifen. Auch in dieser Situation können moving sums verwendet werden, wie in zwei methodischen Arbeiten gezeigt wird, die 1978 und 1980 in der Zeitschrift Technometrics erschienen sind. Für die Encyclopedia of Statistical Sciences von Kotz und Johnson durfte ich einen Eintrag „Moving Sums (MOSUM)"verfassen.

Werner Müller: Es gibt immer noch Zitate davon, das sind so long runner.

Peter Hackl: Diagnostik von Regressionsmodellen in der Anwendung von ökonomischen Fragestellungen, Tests auf Strukturbruch, Kontroll-Karten in der Prozesskontrolle etc. sind auch Themen, die von enormer Relevanz für die Anwendung sind.

Werner Müller : Hast du dann schon begonnen die Arbeiten mit deinen schwedischen Freunden?

Peter Hackl: In Schweden hat partial least squares (PLS) Regression eine lange Tradition. Herman Wold hat die zugrunde liegende Idee zum Modellieren von Relationen zwischen nicht-beobachtbaren Variablen entwickelt. Diese sogenannten structural equation models werden typischerweise in den Sozialwissenschaften verwendet. Mein Befassen mit PLS Regression ist nicht so weit entfernt von meinem Interesse für die Diagnostik von Regressionsmodellen. Mit Anders Westlund, einem Schüler von Herman Wold, und anderen Kollegen vom Institut für Wirtschaftsstatistik der Handelshögskolan in Stockholm sind einige Arbeiten entstanden, in denen Modelle entwickelt werden, die beispielsweise zum Schätzen von customer satisfaction verwendet werden können. Entsprechende Modelle werden in Schweden und einigen anderen Ländern verwendet, um jährliche nationale customer satisfaction Indikatoren zu schätzen.

Werner Müller: Du hast mit dieser Gruppe eine enge Beziehung, du warst dann oft an der Handelshögskolan.

Peter Hackl: Ja, ich habe einige Male das Stockholmer Statistikinstitut besucht. In einem gemeinsamen Projekt mit dem Institut für Marketing der WU haben wir auch für Österreich ein Kundenzufriedenheits-Barometer entwickelt und in einer empirischen Studie über die großen Einzelhandelsketten Spar, Billa und Co ausprobiert.

Werner Müller: Und die Kollaborationen waren so erfolgreich, dass du mit dem Ehrendoktor gewürdigt wurdest.

Peter Hackl: Die Zusammenarbeit mit dem Stockholmer Statistikinstitut hat in den 70er Jahren begonnen, und wir haben eine Reihe von gemeinsamen Forschungs- und Publikations-Projekte durchgeführt. In der zweiten Hälfte der 1980er Jahre war ich Koordinator einer internationalen Arbeitsgruppe Statistical Analysis and Forecasting of Economic Structural Change der IIASA in Laxenburg. In diesem Zusammenhang hat die Handelshögskolan eine internationale Konferenz veranstaltet; ein Ergebnis der Konferenz ist der 1991 bei Springer erschienene Band „Economic Structural Change: Analysis and Forecasting"mit Anders Westlund und mir als Herausgeber. Wir waren auch GastHerausgeber einer Special Section des International Journal of Forecasting zum Thema „Forecasting in the Manufactoring Industry“, erschienen 1996, und eines Special Issue der Zeitschrift Total Quality Management zum Thema „Customer Satisfaction: Theory and Measurement", das 2000 erschien. Das Ehrendoktorat der Handelshögskolan wurde mir 1996 verliehen.

Werner Müller: Gut, das waren die 80-iger und dann ist doch die Situation entstanden, wo ich auch persnlich betroffen war, dass dir die Möglichkeit eingeräumt wurde, eine eigene Abteilung zu gründen. Es war, glaube ich, nicht ganz friktionsfrei, und dann aber doch erfolgreich. Ich durfte dein Assistent werden, Anfang der 90-iger Jahre. Es war ja eine kleine Abteilung, die erst später größer geworden ist, aber am Anfang waren wir nur zu 
zweit plus dem Sekretariat und es ist uns ganz gut gelungen die Ökonometrieausbildung an der Wirtschaftsuniversität wieder auf neue Beine zu stellen. Was für mich recht überraschend war, ich bin ja vom IHS gekommen, dass das praktisch nicht vorhanden war.

Peter Hackl: Ja, das war wirklich merkwürdig. Es war in dieser Zeit im Curriculum der Volkswirte gar nicht vorgesehen, dass sich die Studierenden mit quantitativen Methoden vertieft auseinander setzen. Als Vizestudiendekan für Evaluierung, eine Funktion, die ich an der WU zwischen 1995 und 2000 ausübte, haben wir Verfahren entwickelt und systematisch dafür eingesetzt, nicht nur die didaktischen Leistungen der Lehrenden, sondern auch die Lehrprogramme zu bewerten und Verbesserungspotentiale aufzuzeigen. In diesem Rahmen haben wir auch das Curriculum des Volkswirtschaftsstudiums bewertet. Dabei haben wir mit Volkswirten gesprochen, die schon Jahre in der Berufspraxis stehen, beispielsweise in der ÖNB. Dabei wurde uns gesagt, das größte Handicap der Absolventen von der WU sei, dass sie keine Ausbildung in Ökonometrie bekommen. Da gab es wirklich eine Marktlücke. Unsere Abteilung für Wirtschaftsstatistik hat eine Einführung in die Ökonometrie seit den 80er Jahren angeboten; die von den Studierenden ganz gut angenommen worden ist. Ich habe den Studierenden auch ein Skriptum zur Verfügung gestellt, das die Basis meines Buches „Einführung in die Ökonometrie“war, das bei Pearson Studium verlegt und 2012 in zweiter Auflage erschienen ist.

Werner Müller: Dann in den 90-iger Jahren, da war ich auch am Rande beteiligt, ist dann deine Kollaboration mit Valerii Fedorov und dein Interesse an der Versuchsplanung entstanden. Wir haben ja auch ein paar papers gemeinsam verfasst, die vielleicht nicht sonderlich erfolgreich waren, wenn die Zahl der Zitierungen oder so ansieht, aber immerhin das Buch, das am Rande dieser Aktivität entstanden ist, wird sehr gut angenommen und hat eine hohe Zitationsrate.

Peter Hackl: Das geringe Interesse an den Aufsätzen hat wohl damit zu tun, dass die Anwendungssituationen, etwa aus dem Bereich der Biometrie, für die uns Daten zur Verfügung standen, eher exotisch waren. Unser Buch „Model-Oriented Design of Experiments", das du angesprochen hast, ist 1997 erschienen und wird bis heute häufig zitiert. Ausgangspunkt für das Buch waren Unterlagen, die Valerii in seiner Vorlesung als Gastprofessor unseres Instituts den Studierenden zur Verfügung gestellt hat.

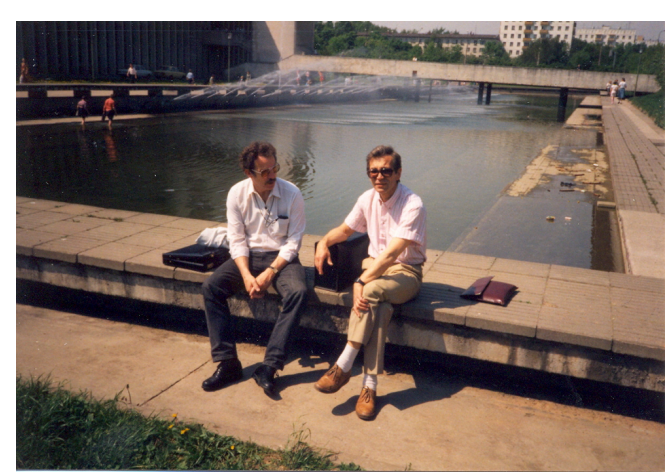

Mit Valerii Fedorov in Moskau 1989.

Werner Müller: Jetzt ist es total in. Mit Versuchsplänen für klinische Studien beschäftigen sich heute ganze Institute damit, aber das Thema würde nun zu weit führen.

Kommen wir in die Mitte der 90-iger Jahre, deine Tätigkeit als Präsident der Österreichischen Statistischen Gesellschaft. Du warst ja mehrere Perioden Präsident und hast die Präsidentschaft in einer schwierigen Phase übernommen, die nicht ganz friktionsfrei war. Vielleicht kannst du schildern, wie die Ausgangslage war.

Peter Hackl: Das war wirklich eine ungute Geschichte. Das Statut der ÖSG hat vorgesehen, dass die Statistische Gesellschaft von zwei Vorsitzenden geführt wird, von denen einer der Präsident des Statistischen Zentralamtes ist, der andere ein Vertreter der akademischen Statistik. An sich eine exzellente Idee, die seit Bestehen der ÖSG so konzipiert war, deren gutes Funktionieren aber von den handelnden Personen abhängt. Ab dem Jahr 1995 war ich gemeinsam mit Präsident Bader einer der Vorsitzenden der ÖSG. Schon in den Jahren davor ist die Zusammenarbeit im Vorstand der ÖSG schwierig geworden, 
so dass eine Umstrukturierung angedacht wurde, die in das derzeit bestehende Statut mündete. Die damals eingeführte Arbeitsteilung zwischen Amtlicher, Akademischer und Angewandter Statistik und die Rotation der Präsidentschaft zwischen Vertretern dieser Bereiche haben sich inzwischen sehr gut bewährt. Sie hat zu einer stärkeren Einbindung der Angewandten Statistik und einem gewachsenen Interesse der Akademischen Statistik an Fragen der Angewandten und Amtlichen Statistik geführt. Das war und ist an der seit damals gewachsenen Zahl der Arbeitskreise und Veranstaltungen und an der sehr erfolgreichen Einbindung der Studierenden in die Aktivitäten der ÖSG ablesbar. Ich möchte in diesem Zusammenhang zwei Namen von Personen nennen, die für den Übergang und neuen Aufschwung der ÖSG Entscheidendes geleistet haben: Wilfried Grossmann ist das Funktionieren der ÖSG in der schwierigsten Phase zu verdanken, und Michaela Denk hat den Arbeitskreis Junge Statistik sehr erfolgreich aufgebaut.

Werner Müller: Viele Dinge von denen wir jetzt noch zehren in unserer Struktur und Aktivitäten ist ja damals eingeführt wurde, wie Statistiktage, Förderpreise und all diese Dinge.

\section{Peter Hackl:}

Es war sicher an der Zeit, neue Ideen einzubringen. Was wollen die ÖSG-Mitglieder, wie kann man die Mitglieder mehr einbeziehen, wie kann man mehr Arbeitsgruppen haben, die gezielt ihre Interessen verfolgen. Die Statistischen Kolloquien, den methodischen Arbeitskreis, haben Peter Bauer und ich Anfang der 70-iger Jahre vorgeschlagen, und in den späten 70-iger Jahren sind weitere Arbeitskreise entstanden.

Nach der Umstrukturierung der ÖSG haben wir die Zahl der Arbeitskreise massiv ausgeweitet. Jeden entsprechenden Vorschlag haben wir gefördert und unterstützt; vieles ist auch wieder eingeschlafen. Aber generell hat sich die Idee gut bewährt, und es ist damals viel entstanden. Auch die Zeitschrift der ÖSG, die Österreichische Zeitschrift für Statistik, haben wir auf neue Beine gestellt.

Matthias Templ: Was hat schließlich bewegt zur Statistik Austria zu wechseln und dort die Führung als Fachstatistischer Generaldirektor zu übernehmen?

Peter Hackl: Es war nicht wirklich angestrebt von mir. In meiner Zeit als Präsident der Statistischen Gesellschaft wurden die Beitrittsverhandlungen mit der EU geführt. Da spielte die Amtliche Statistik eine zentrale Rolle: Die Art und Weise, wie ein Land gesehen wird, wird maßgeblich durch Statistiken geprägt, vor allem die Wirtschaftsstatistiken und die makroökonomischen Kennzahlen des Landes. Die Harmonisierung der österreichischen Statistiken mit dem Acquis Communautaire, dem Kompendium der von den Mitgliedstaaten zu liefernden Statistiken, war für das Statistische Zentralamt keine einfache Aufgabe. Komplikationen und vor allem Verzögerungen nahmen Ausmaße an, die schließlich sogar von den Medien breit berichtet und sehr negativ kommentiert wurden. Schließlich hat die Politik dann beschlossen, das Statistikgesetz zu ändern und damit auch die ganze organisatorische Basis der Amtlichen Statistik. Die Statistische Gesellschaft hat sich in die entsprechenden Überlegungen und Bemühungen eingebracht. Im schließlich beschlossenen Statistikgesetz war vorgesehen, dass es zur Statistik Austria, wie das Statistikamt ab 2000 genannt wurde, auch einen Beirat geben soll, den Statistikrat. Er ist, verglichen mit den Beiräten anderer Statistikämter, durch seinen jährlichen Bericht an Parlament und Regierung ein ziemlich mächtiges Gremium. Unter anderem ist vorgesehen, dass ein Vertreter der Wissenschaft, der im Fach Statistik habilitiert ist, im Statistikrat einen Sitz hat. Allzu viele Vertreter der akademischen Statistik mit Affinität zur Amtlichen Statistik gab es nicht, und so hatte ich die Ehre, für den Zeitraum 2000 bis 2004 in den Statistikrat bestellt zu werden. In dieser Zeit musste ich mich naturgemäßsehr massiv mit der Amtlichen Statistik befassen. Die Kompetenzen des Statistikrats sind sehr breit angelegt: Kernangelegenheiten betreffen das Jahresprogramm, den Jahresbericht und den jährlichen Bericht an Parlament und 
Regierung; daneben hat sich das Gremium mit verschiedensten anderen Fragen befasst, zentral darunter um Fragen zur Qualität der statistischen Produkte. Als die Amtszeit meines Vorgängers, Ewald Kutzenberger, zu Ende ging, wurde ich vom Bundeskanzleramt eingeladen, eine Bewerbung abzugeben. Nach einer längeren Nachdenkphase habe mich schließlich beworben. Das Ergebnis des Auswahlverfahrens ist euch ja bekannt.

Matthias Templ: Für die methodische Statistik war es für mich persönlich ein großer Glücksfall, weil von dir der Kontakt zur Methodik intensiv gesucht wurde. Im Speziellen warst du auch interessierst modernere Methoden in der Statistik anzuwenden und zu entwickeln. Aufgrund der Freiheiten die wir von dir bekommen haben, konnten wir in der Zeit einige neue Entwicklungen vorantreiben von denen wir heute noch profitieren. Ich stelle es mir trotzdem schwierig vor neue Wege zu beschreiten, weil man immer die Reibungsstelle ist. Sobald man neue Sachen umsetzen will stößt man oft auf Widerstand und Unverständnis. Aber du bist immer sehr pragmatisch vorgegangen und bist jedem immer in respektvoller Weise begegnet.

Peter Hackl: Ja, Respekt gegenüber anderen Menschen habe ich schon zu Hause gelernt. Auf der anderen Seite ist der Spielraum für neue Entwicklungen in dieser Funktion beschränkt. Ein Thema bei dem, im Nachhinein gesehen, wahrscheinlich mehr hätte passieren sollen, das aber ganz schwierig war, war der IT-Bereich. Die Statistik Austria hat damals Groß-IT verwendet; das war sehr teuer und sehr unbeweglich mit dem Vorteil einer hohen Sicherheit der Daten. Andererseits konnten viele Chancen der TechnologieEntwicklung nicht genutzt werden. In diesem Bereich habe ich sicherlich nicht alle Erwartungen erfüllen können. Beispielsweise hat sich Josef Richter im Statistikrat massiv für Metadaten-gesteuerte statistische Prozesse eingesetzt hat, ein Thema, das ganz richtig liegt, das man aber nicht so leicht hinbekommt.

Matthias Templ: Das ist nach wie vor ein Dauerthema.

Peter Hackl: Jetzt kommt es von der anderen Seite, weil von Eurostat das generic statistical business process model (GSBPM) forciert wird, ein Konzept für den statistischen Prozess, das für alle statistischen Produkte anwendbar sein soll. Es wird in vielen europäischen Statistikämtern schrittweise implementiert. Wir haben mit einem Entwurf für ein Metadaten-Repositorium angefangen. Die Umsetzung wäre eine riesige Investition geworden, und es war nicht abzusehen, was herauskommt. Der nächste Schritt des Projektes, die Steuerung der statistischen Prozesse, wäre ein Unternehmen gewesen, an dem einige andere Statistikämter gescheitert sind. Zu radikale Änderungen waren für die Statistik Austria nicht ohne weiteres möglich; man muss auch die Beschränkungen akzeptieren. Andererseits wurde im Rahmen der Strategie 2006-2010 eine große Zahl von innovativen Projekten durchgeführt. Vor allem ist hier der Umstieg von traditionellen Volks-, Gebäude- und Betriebszählungen auf eine registergestützte Zählung zu nennen, ein Vorhaben, dessen Konzeption und Realisierung vor allem dem leider so früh verstorbenen Peter Findl zu verdanken ist. Eine Auswahl weiterer innovativer Projekte: die Neukonzeption der Website, die Entwicklung des regionalstatistischen Online-Atlas, der Aufbau einer Arbeitsmarktstatistik-Datenbank, die Einrichtung des Forschungsschwerpunktes Plausibilitätsprüfung und Imputation, die Einrichtung eines Web-Portals für Studierende, die Kooperation mit Universitäten und anderen Forschungseinrichtungen, die Beteiligung an internationalen Forschungsprojekten wie dem FP7 Projekt Advanced Methods for Laeken Indicators (AMELI) und dem CENEX-Projekt ISAD, die Etablierung der vertieften Kooperation mit den Nationalen Statistischen Ämtern der Nachbarländer Tschechien, Slowakei, Ungarn und Slowenien. Insgesamt ist die Statistik Austria in dieser Zeit, vor allem nach der Umstrukturierung in der Zeit meines Vorgängers Ewald Kutzenberger, unter den europäischen Statistikämtern sehr gut dagestanden.

Matthias Templ: Ich kann mich an den Peer Review Bericht aus dem Jahr 2008 erinnern, welcher sehr positiv ausgefallen ist. 
Peter Hackl: Aus den Bewertungen der peer review Berichte aus 2007 und 2008 kann man ein Ranking der Statistikämter ableiten: Die Statistik Austria hatte darin gemeinsam mit Finnland den ersten Platz. Ich glaube, auch jetzt steht die Statistik Austria recht gut da. In der Sozialstatistik hat Peter Findl tüchtigen, jungen Leuten die Chance gegeben, wirklich schöne Sachen zu machen und aufzubauen. Auch andere Bereiche haben sich sehr gut entwickelt.

Matthias Templ Du hast vom GSBPM gesprochen, das ist eines von den Modellen, welches momentan sehr stark herumgeistert. Findest du es wichtig?

Peter Hackl: Ich kann es nicht wirklich beurteilen, dazu müsste man mehr mit den Details vertraut sein. Aber ich habe mir ein paar Websites von Statistikämtern angeschaut und die Ämter von Deutschland, Bulgarien, Zypern und Ungarn als peer kennen gelernt. Alle reden vom GSBPM, das ist ja klar, schließlich gibt es eine Vorgabe von Eurostat. Alle denken darüber nach, wie das GSBPM implementiert werden kann. Ein paar sind schon weiter; die Deutschen haben schon einige Module, wie zur Erhebung und zum Validieren und Editieren der Daten. Ich glaube, dass es nirgends sehr rasch gehen wird, weil das Implementieren erstens einen großen Aufwand erfordert und zweitens das GSBPM sehr komplexe Teile enthält. Der Output kann auch von sehr unterschiedlicher Qualität sein. Ob das GSBPM wirklich das Gelbe vom Ei ist, traue ich mich nicht zu beurteilen. Das Konzept sieht vernünftig aus. Es umfasst die Aktivitäten des Statistikers vom Design des statistischen Produkts bis zur Publikation und dem Archivieren. In den meisten statistischen Produktionen braucht man nur Teile davon. Ob man das alles so umsetzen kann, ob es Interfaces gibt zwischen den einzelnen Modulen, die es erlauben, das System wie aus einem Guss zu verwenden, wird man sehen. Meines Wissens gibt es noch nirgends ein komplettes System von der Art des GSBPM. Aber ich glaube, dass es eine gute Sache ist, dass darüber nachgedacht wird, wie man den Statistikprozess systematischer machen kann. Es ist keine Frage, dass sehr viel Potenzial an Vereinfachung fr den laufenden Betrieb vorhanden ist, und eine bessere Kosteneffektivität erreicht werden kann und dringend notwendig ist.

Matthias Templ: Du hast schon erwähnt, dass einem Peer-review als Generaldirektor der Statistik Austria unterzogen warst. Jetzt bist du selbst Peer-Reviewer.

Peter Hackl: Ja, ich war eingeladen, in den genannten Ländern am peer review mitzuwirken. Es gibt eine neue Runde der peer reviews, die 2014/15 läuft. Man möchte wissen, ob die Empfehlungen umgesetzt wurden, die in der ersten Runde ausgesprochen wurden, wie weit die Ämter in der Umsetzung des Code of Practice sind, und, ein neues Thema, wie gut die Koordinierung aller mit der Amtlichen Statistik eines Landes befassten Institutionen funktioniert. Wird z.B. die Migrationsstatistik in einem Ministerium oder die Statistiken aus dem Umweltbereich von einer eigenen Anstalt produziert, so sollten, das ist die Vorstellung der EU, die verantwortlichen Stellen ebenfalls die Prinzipien des Code of Practice anwenden. Die neuen peer review Berichte werden auch dazu Empfehlungen für die Länder machen.

Matthias Templ: Wäre das eine Empfehlung, dass die Statistik Austria auch Umweltdaten analysieren sollte, weil es Auswirkungen auf die Gesellschaft hat.

Peter Hackl: In Österreich gibt es schon eine gute Zusammenarbeit mit dem Umweltbundesamt. In meiner Zeit ist da sehr viel gemeinsam besprochen worden, und so viel ich weiß, werden manche Statistiken aus dem Bereich Umwelt von der Statistik Austria produziert. Beim peer review Bericht geht es vor allem um die Koordinierungsfunktion, es geht um die professionelle Unabhängigkeit, die sicherstellt, dass alle mit Amtlicher Statistik befassten Institutionen Weisungsfreiheit genießen, und um weitere Punkte wie methodische Zusammenarbeit und Hilfestellung, Schulung, etc. Der Code of Practice hat sich in seiner Umsetzung weiterentwickelt, und daher hat man befunden, dass eine 
neue Runde peer reviews gemacht werden soll. Die Arbeit der peers ist naturgemäßsehr interessant.

Werner Müller: Auch beim Unterrichten bist ja noch aktiv. Vor wenigen Jahren ist eine neue Ausgabe von deinem Ökonometrielehrbuch erschienen, du unterrichtest Ökonometrie noch.

Peter Hackl: Ja, ich halte für die Studierenden des PhD-Programms in Volkswirtschaft an der Masaryk Universität in Brno eine Pflichtlehrveranstaltung ab. Für einige wenige Studierende, die es genauer wissen wollen, gibt es jeweils im Sommersemester auch einen vertiefenden zweiten Teil. Ich halte diese Vorlesung recht gerne. Mein Ökonometriebuch kann ich leider nicht verwenden, da ein englisches Textbuch zu verwenden ist. Die Studierenden können ganz gut Englisch, Deutsch aber nur vereinzelt.

Matthias Templ: Ich lese in deinem Lebenslauf noch von einigen interessanten Orten, welche du als statistischer Experte besucht hast, wie in Abu Dhabi, in ... klingt abenteuerlich.

Peter Hackl: Meine Tätigkeiten waren - leider oder Gott sei Dank - zumindest bisher nicht abenteuerlich. Nach meiner Tätigkeit für die Statistik Austria wurde ich zu verschiedenen Beratungsarbeiten eingeladen. Auf meiner Liste finden sich - neben Abu Dhabi die Ukraine, Bulgarien, Albanien, Georgien, Azerbaijan, Palestina, und andere. Meist geht es um Themen der Amtlichen Statistik, oft um das Bewerten des Statistikamtes. Das Statistical Centre of Abu Dhabi (SCAD) ist ziemlich jung, aber interessant. Das Management ist sehr ehrgeizig, aber die Voraussetzungen sind schwierig. In Abu Dhabi gibt es kein Statistikstudium. Das SCAD hat in wichtigen Bereichen ausländische Experten, natürlich begleitet von einem Chef, der ein Emirati ist. Abu Dhabi hat exzellente Manager, vor allem in der Ölindustrie, die Betriebsführung verstehen sie sehr gut, und auch im SCAD spielen Strategie und Managementtechniken eine wichtige Rolle. Ich hatte im Herbst 2012 die Ehre, eine Strategie, den sogenannten Masterplan, zu schreiben, der die wichtigen Entwicklungspotentiale aufzeigt und einen Aktionsplan für die kommenden Jahre vorschlägt. Seither gehöre ich auch dem International Advisory Comittee des Generaldirektors des SCAD an, fünf pensionierte Amtsleiter, die halbjährlich zu ein- oder zweitägigen Beratungen zusammen kommen. Noch ein Wort zum Ehrgeiz: Das SCAD wird die 2016-Konferenz der International Association of Official Statistics veranstalten. Meine Beratungsarbeiten sind sehr interessante Aufgaben. Man sieht, wo ein Statistikamt seine Probleme hat und wie es sich entwickeln kann. Wenn man da mithelfen kann, ist das sehr interessant.

Matthias Templ: Ist es nicht zu heiß, einen Marathon in Abu Dhabi zu laufen?

Peter Hackl: Das habe ich nicht vor, und ich bin gar nicht sicher, ob in Abu Dhabi einer veranstaltet wird. Ich bin viermal den Marathon gelaufen, habe aber dann Probleme mit beiden Knien gehabt. Seither bin ich nur mehr Halbmarathons gelaufen. Ich laufe aber regelmäßig mehrmals die Woche, vor allem aus Gesundheitsgründen, und glaube, dass sich das auch bezahlt macht.

Matthias Templ: Die Statistik ist derzeit mit großen Herausforderungen konfrontiert. Was sind die Kernkompetenzen der Statistik und wo gibt es eine Abgrenzung zu anderen Fächern. Stichwort Big Data. Hast du eine Vision wie sich die Statistik in Zukunft entwickeln soll?

Peter Hackl: Ich finde, dass sich das Ansehen und die Akzeptanz der Statistik in der Öffentlichkeit in den letzten Jahren sehr gut entwickelt hat, dass es aber nach wie vor riesige Defizite gibt, was die Rolle der Statistik im öffentlichen Leben spielt. Ich möchte das an einem Beispiel ausführen. Kürzlich habe ich einen Bericht im ORF Radio über die zunehmende Anzahl von resistenten Keimen gehört, die in Krankenhäusern immer mehr zum Problem werden. Eine ganz probate Möglichkeit, mit resistenten Keimen 
umzugehen, wären Statistiken über die Zahl der Problemfälle, gegliedert nach Krankenhäusern, die auch noch entsprechend detailliert werden können. Solche Statistiken wären wichtige Hinweise für die Gesundheitspolitik, aber auch für Patienten; sie wären darüber hinaus Basis für das Verstehen der Ausbreitung dieser Keime und für die Planung von notwendigen Maßnahmen. Die Basisdaten gibt es zumindest teilweise. Warum nicht die Statistiken? Eine Linzer Expertin für Hygiene im Krankenhaus erklärte in dieser ORF-Sendung, es wäre gefährlich, der Öffentlichkeit diese Information zu geben: Journalisten würden Horrorgeschichten daraus machen, und die Menschen würden durch diese Fakten verunsichert und verängstigt. Man versteht: Es gibt keinen Druck, diese Statistiken zu produzieren, und der Grund dafür ist vermutlich, dass die Wichtigkeit eines faktenbezogener Umgangs mit der Realität von den Verantwortlichen gar nicht verstanden wird. Ein Beispiel dafür, dass in Österreich das Bereitstellen von Statistiken bisweilen geradezu böswillig verhindert wird, ist das Verweigern detaillierter Ergebnisse der österreichischen PISA-Daten. Ergebnisse nach Regionen und nach Schultypen würden wesentlich zur Versachlichung der laufenden Schuldiskussion beitragen. Ein nicht faktenbezogener Umgang mit der Realität führt zu schlechten und teuren Entscheidungen. In Zeiten der Big Data ist das Anliegen noch viel dringender. Das Streichen des Amtsgeheimnisses für die öffentliche Verwaltung wäre ein wichtiger Schritt, um den Umgang mit Informationen in der Öffentlichkeit zu verbessern. Die Statistik Austria hat in letzten Jahren sehr viel dazu beigetragen. Stichwörter aus meiner Zeit sind hier: neue Website, regionalstatistischer Online-Atlas, erleichterter Zugriff auf Daten für die Forschung, flächendeckende Metadaten.

Big Data ist vor allem ein riesen $\mathrm{Hy}-$ pe. Symptomatisch ist, dass es noch keine tragfähige Definition für big data gibt. Die Verfügbarkeit von ungeheuer großen Datenmengen ist zweifelsohne eine Herausforderung für Statistiker.

Data Science ist ein Bereich, in dem die Statistiker aufpassen müssen, dass sie das Feld nicht zu sehr den Informatikern überlassen. Problematisch ist dabei auch, dass Informatiker inhaltlichen Beschränkungen oft nicht die notwendige Bedeutung beimessen; ein weiterer Grund dafür, dass sich Statistiker in diesen Bereich einbringen.

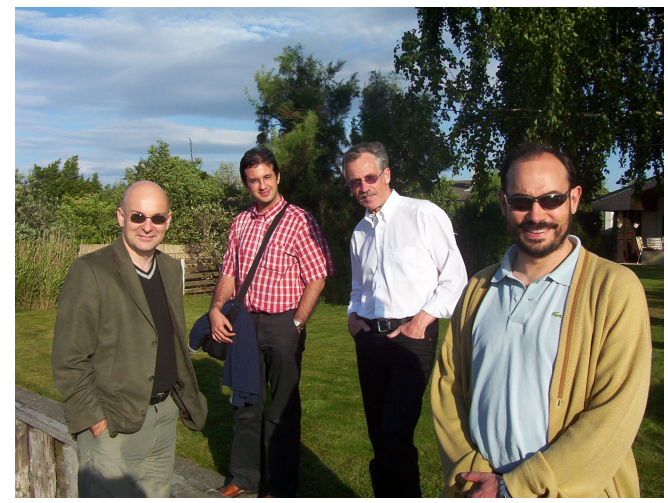

Mit Werner Müller, Raul Martín-Martín und Jesus Lopéz-Fidalgo (von links) in Neusiedl/See 2005.

Werner Müller: Dies ist ja mittlerweile widerlegt, das das so gut funktioniert.

Peter Hackl: Für die Amtliche Statistik können alternative Datenquellen wie das Internet eine sehr brauchbare Ergänzung zu Daten aus Erhebungen und administrativen Quellen sein. Inzwischen gibt es im Bereich der Amtlichen Statistik einige konkrete Anwendungen der Nutzung von solchen Daten; dabei wird auch gerne von Big Data gesprochen. Beispiele sind die Verwendung von Ticketpreisen von Fluglinien aus dem Internet für den Verbraucherpreisindex, die Verwendung von Daten von Telefonprovidern in der Tourismusstatistik, oder von remote sensing Daten in der Agrarstatistik. Diese Aufzählung zeigt auch, wie heterogen die sogenannten big data sind. Die Nachhaltigkeit dieses Begriffs ist jedenfalls zu bezweifeln.

\section{Matthias Templ, Werner Müller: Danke für das Gespräch.}

Die Interviewer bedanken sich herzlich bei Gabriele Mack-Niederleitner (Johannes Kepler Universität Linz) für die Transkription, und bei Klára Hrŭzová für die Konvertierung in das AJS Format. 


\section{Affiliation:}

Peter Hackl

Wohnhaft im Burgenland

Werner Müller

Johannes Kepler University Linz

Department of Applied Statistics

Altenberger Straße 69

A-4040 Linz, Austria

E-mail: werner.mueller@jku.at

Matthias Templ

Vienna University of Technology \&

Statistik Austria

A-1040 Vienna, Austria

E-mail: matthias.templ@gmail.com

\section{Austrian Journal of Statistics}

published by the Austrian Society of Statistics

Volume 44

October 2015 http://www.ajs.or.at/

http://www.osg.or.at/

Submitted: 2015-03-20

Accepted: 2015-04-20 